\title{
THE AXISYMMETRIC BOUSSINESQ-TYPE PROBLEM FOR A HALF-SPACE UNDER OPTIMAL HEATING OF ARBITRARY PROFILE
}

\author{
J. ROKNE, B. M. SINGH, R. S. DHALIWAL, and J. VRBIK
}

Received 25 August 2003

\begin{abstract}
A solution of the axisymmetric Boussinesq-type problem is derived for transient thermal stresses in a half-space under heating by using the Laplace and Hankel transforms. An analytical method is developed to predict the temperature field that satisfies the prescribed mechanical conditions. Several simple shapes of punches of arbitrary profile are considered and an expression for the total load is derived to achieve penetration. The numerical results for the temperature and the total load on the punch are shown graphically.
\end{abstract}

2000 Mathematics Subject Classification: 44A10, 45B05, 45F10, 74B05.

1. Introduction. The problem of determining (within the terms of the classical theory of elasticity) the distribution of stresses within an elastic half-space when it is deformed by a normal pressure against its boundary by a rigid punch seems to have been first considered by Boussinesq (see $[1,6]$ ). After the publication of the Boussinesq solution, several alternative solutions were derived, an excellent account of which is given in the books by Galin [2] and Sneddon [4] as well as in the paper by Sneddon [5]. In this paper, we consider the analysis of a mixed boundary value problem for a halfspace in a transient thermoelasticity under the prescribed mechanical boundary condition. We consider the thermal stresses produced in the semi-infinite elastic medium $z>0$ bounded only by the plane $z=0$. The solid is supposed to be deformed by being pressed against a perfectly rigid solid of revolution of prescribed shape whose axis of revolution coincides with the $z$-axis of the coordinate system (and hence is normal to the boundary plane). It is obvious from the axial symmetry that the strained surface of the elastic medium will fit the rigid body over the part between the lowest point and a certain circular section.

In the formation of the problem, we assume that, on the one hand, the initial condition is that the temperature is zero at time zero and, on the other hand, the mechanical problem is defined by conditions (2.4). The main results of the paper are that the resultant force applied on the punch and the temperature distribution in the half-space are obtained. The analysis for finding the distribution of temperature and stresses in this type of problems has undergone marked development in connection with such problems as those arising during the design of steam and gas turbines and nuclear reactors.

The mathematical analysis is developed by using the Laplace and Hankel transforms and the solution of the problem is reduced to dual integral equations of Bessel functions. The solution of the dual integral equations is reduced to a Fredholm integral 
equation of the second kind. The Fredholm integral equation is solved numerically and the numerical results for the resultant force applied to a punch of arbitrary profile are obtained and shown graphically for two specific profiles.

2. Thermal stress. Let $(r, \theta, z)$ be the polar cylindrical coordinates. By using the thermoelastic potential function $\Phi$ and Love's function $L$ for the axisymmetric problem, nonzero displacements $u_{i}$ and stresses $\sigma_{i j}$ are given by

$$
\begin{aligned}
u_{r} & =\Phi_{, r}-L_{, r z}, \\
u_{z} & =\Phi_{, z}+2(1-v) \Delta^{2} L-L_{, z z}, \\
\frac{\sigma_{r r}}{2 \mu} & =\Phi_{r r}-\Delta^{2} \Phi+\left(v \Delta^{2} L-L_{, r r}\right)_{, z}, \\
\frac{\sigma_{\theta \theta}}{2 \mu} & =\frac{1}{r} \Phi^{\prime} r-\Delta^{2} \Phi+\left(v \Delta^{2} L-r^{-1} L_{, r}\right)_{, z}, \\
\frac{\sigma_{z z}}{2 \mu} & =\Phi^{\prime} z z-\Delta^{2} \Phi+\left[(2-v) \Delta^{2} L-L_{, z z}\right]_{, z}, \\
\frac{\sigma_{r z}}{2 \mu} & =\Phi_{r z}+\left[(1-v) \Delta^{2} L-L_{, z z}\right]_{, r},
\end{aligned}
$$

where $\mu$ is Lame's constant, $v$ is the Poisson ratio, $\Delta^{2}=\partial^{2} / \partial r^{2}+(1 / r)(\partial / \partial r)+\partial^{2} / \partial z^{2}$, and the comma denotes partial differentiation with respect to a variable.

The thermoelastic potential function $\Phi$ and Love's function $L$ must satisfy the following differential equations:

$$
\begin{gathered}
\Delta^{2} \Phi=K T, \quad K=\left(\frac{1+v}{1-v}\right) \alpha, \\
\Delta^{4} L=0,
\end{gathered}
$$

where $T$ is the temperature change and $\alpha$ is the coefficient of linear thermal expansion.

The boundary conditions for the problem are

$$
\begin{aligned}
\sigma_{r z}(r, 0, t) & =0, \quad 0<r<\infty, \\
\sigma_{z z}(r, 0, t) & =0, \quad a<r<\infty, \\
u_{z}(r, 0, t) & =u_{0} f(r) g(t), \quad 0<r<a,
\end{aligned}
$$

where $u_{0}$ is a constant and $f(r)$ and $g(t)$ are the prescribed functions with respect to position $r$ and time $t$, respectively.

We introduce the Laplace transform $u_{z}^{*}(r, z, p)$ of the function $u_{z}(r, z, t)$ with respect to $t$ by

$$
u_{z}^{*}=u_{z}^{*}(r, z, p)=\int_{0}^{\infty} u_{z}(r, z, t) e^{-p t} d t
$$

where $p$ is the Laplace parameter. Performing the Laplace transform on (2.3), the Love function $L^{*}$ in the transform domain may be expressed by

$$
L^{*}=K \int_{0}^{\infty} s^{-2}[1+s z] e^{-s z} A(s, p) J_{0}(r s) d s,
$$


where $J_{v}(r s)$ denotes the Bessel function of first kind of order $v \geq 0$ and $A(s, p)$ is an unknown function to be determined from the boundary conditions. The displacement $u_{z}^{*}$ and stresses $\sigma_{z z}^{*}, \sigma_{r z}^{*}$ in the transform domain are given by

$$
\begin{aligned}
u_{z}^{*} & =\frac{\partial \Phi^{*}}{\partial z}+K \int_{0}^{\infty}[(4 v-3)-s z] A(s, p) e^{-s z} J_{0}(r s) d s \\
\frac{\sigma_{z z}^{*}}{2 \mu} & =\Phi_{, z z}^{*}-\Delta^{2} \Phi^{*}+\int_{0}^{\infty}\left[s^{2} z+2 s(1-v)\right] A(s, p) e^{-s z} J_{0}(r s) d s, \\
\frac{\sigma_{r z}^{*}}{2 \mu} & =\frac{\partial^{2} \Phi^{*}}{\partial r \partial z}-\int_{0}^{\infty} s[-(1-v)(1+s z)+v(1-s z)] A(s, p) e^{-s z} J_{0}(r s) d s,
\end{aligned}
$$

where the displacement $u_{r}^{*}$ and stresses $\sigma_{r r}^{*}, \sigma_{\theta \theta}^{*}$ are omitted.

Next, an axisymmetrical fundamental equation for the transient heat condition without heat generation is

$$
k \Delta^{2} T=T_{, t}
$$

where $k$ is the thermal diffusivity. When the initial temperature is zero, the Laplace transform of (2.8) is

$$
k \Delta^{2} T^{*}=p T^{*}
$$

For this problem, the general solution of (2.9) may be taken as

$$
T^{*}=\int_{0}^{\infty} D(s, p) e^{-\left(s^{2}+p / k\right)^{1 / 2} z} J_{0}(r s) d s .
$$

Substituting (2.10) into (2.2) $)_{1}$ in the transform domain, the expression for the thermoelastic potential function is given by

$$
\Phi^{*}=K \frac{k}{p} \int_{0}^{\infty} D(s, p) e^{-\left(s^{2}+p / k\right)^{1 / 2} z} J_{0}(r s) d s .
$$

Substituting (2.11) into (2.7), we find that

$$
\begin{aligned}
\frac{u_{z}^{*}}{K}= & -\frac{k}{p} \int_{0}^{\infty}\left(s^{2}+\frac{p}{k}\right)^{1 / 2} D(s, p) e^{-\left(s^{2}+p / k\right)^{1 / 2} z} J_{0}(r s) d s \\
& +\int_{0}^{\infty}[(4 v-3)-s z] A(s, p) e^{-s z} J_{0}(r s) d s, \\
\frac{\sigma_{z z}^{*}}{2 \mu K}= & \frac{k}{p} \int_{0}^{\infty} s^{2} D(s, p) e^{-\left(s^{2}+p / k\right)^{1 / 2} z} J_{0}(r s) d s \\
& +\int_{0}^{\infty}\left(z s^{2}+2 s(1-v)\right) A(s, p) e^{-s z} J_{0}(r s) d s, \\
\frac{\sigma_{r z}^{*}}{2 \mu K}= & \frac{k}{p} \int_{0}^{\infty} s D(s, p)\left(s^{2}+\frac{p}{k}\right)^{1 / 2} e^{-\left(s^{2}+p / k\right)^{1 / 2} z} J_{1}(r s) d s \\
& +\int_{0}^{\infty} s[(1-v)(1+s z)-v(1-s z)] A(s, p) e^{-s z} J_{1}(r s) d s .
\end{aligned}
$$


The boundary conditions (2.4) in the Laplace transform domain may be written as

$$
\begin{aligned}
\sigma_{r z}(r, 0, p) & =0, \quad 0<r<\infty, \\
\sigma_{z z}(r, 0, p) & =0, \quad a<r<\infty, \\
u_{z}(r, 0, p) & =u_{0} f(r) g^{*}(p), \quad 0<r<a .
\end{aligned}
$$

Making use of condition (2.15), we find that

$$
D(s, p)=-\frac{p}{k}\left[\frac{(1-2 v) A(s, p)}{\left(s^{2}+p / k\right)^{1 / 2}}\right] .
$$

Making use of (2.18), we find, from (2.12) and (2.13), that

$$
\begin{aligned}
\frac{u_{z}^{*}}{K} & =-2(1-v) \int_{0}^{\infty} A(s, p) J_{0}(r s) d s \\
\frac{\sigma_{z z}^{*}}{2 \mu K} & =\int_{0}^{\infty}\left[2(1-v)-\frac{s(1-2 v)}{\left(s^{2}+p / k\right)^{1 / 2}}\right] s A(s, p) J_{0}(r s) d s .
\end{aligned}
$$

From boundary conditions (2.17) and (2.16), respectively, we find that

$$
\begin{gathered}
\int_{0}^{\infty} C(s, p) J_{0}(r s) d s+\int_{0}^{\infty} K_{2}(s, p) C(s, p) J_{0}(r s) d s=\frac{u_{0} f(r) g^{*}(p)}{2(1-v) K}, \quad 0<r<a, \\
\int_{0}^{\infty} s C(s, p) J_{0}(r s) d s=0, \quad a<r
\end{gathered}
$$

where

$$
\begin{aligned}
C(s, p) & =-\frac{A(s, p)}{K_{1}(s, p)}, \\
K_{1}(s, p) & =\frac{\left(s^{2}+p / k\right)^{1 / 2}}{\left[2(1-v)\left(s^{2}+p / k\right)^{1 / 2}-s(1-2 v)\right]}, \\
K_{2}(s, p) & =K_{1}(s, p)-1 .
\end{aligned}
$$

We use the following representation:

$$
C(s, p)=\int_{0}^{a} \chi(u, p) \cos (s u) d u
$$

On integrating (2.25) by parts, we get

$$
C(s, p)=\left[\frac{\chi(a, p) \sin (s a)}{a}-\frac{1}{s} \int_{0}^{a} \chi^{\prime}(u, p) \sin (s u) d u\right],
$$

where the prime denotes the derivative with respect to $t$. Substituting the expression (2.25) into (2.21), we find that (2.21) is identically satisfied and that (2.20) leads to the following integral equation:

$$
\int_{0}^{r} \frac{\chi(u, p) d u}{\left(r^{2}-u^{2}\right)^{1 / 2}}+\int_{0}^{\infty} K_{2}(s, p) C(s, p) J_{0}(r s) d s=\frac{u_{0} g^{*}(p) f(r)}{2(1-v) K}, \quad 0<r<a .
$$


The above equation is of Abel type and hence its solution may be written in the following form:

$$
\chi(u, p)+\int_{0}^{a} \chi(\nu, p) M(u, v, p) d v=\frac{u_{0} g^{*}(p)}{\pi(1-v) K} \frac{d}{d u} \int_{0}^{u} \frac{r f(r) d r}{\left(u^{2}-r^{2}\right)^{1 / 2}}, \quad 0<t<a,
$$

where

$$
M(u, v, p)=\frac{2}{\pi} \int_{0}^{\infty} K_{2}(s, p) \cos (u s) \cos (v s) d s
$$

and we have used (2.25) and the following result:

$$
\cos (s t)=\frac{d}{d t} \int_{0}^{t} \frac{r J_{0}(s r) d r}{\left(t^{2}-r^{2}\right)^{1 / 2}}
$$

Equation (2.28) is a Fredholm integral equation of the second kind, which can be solved numerically.

3. Formula for the total load on the punch. The total load $P$ on the punch required to produce the above penetration is given by

$$
P^{*}=-2 \pi \int_{0}^{a} r \sigma_{z z}^{*}(r, 0, p) d r
$$

where

$$
\sigma_{z z}^{*}(r, 0, p)=-\frac{1}{r} \frac{\partial}{\partial r} r \int_{0}^{\infty} C(s, p) J_{1}(r s) d s .
$$

Substituting the value of $C(s, p)$ from (2.25) into (3.2), we obtain

$$
\sigma_{z z}^{*}(r, 0, p)=-\frac{1}{r} \frac{\partial}{\partial r} \int_{r}^{a} \frac{u \chi(u, p) d u}{\left(u^{2}-r^{2}\right)^{1 / 2}}, \quad 0<r<a .
$$

Making use of (3.1) and (3.3), we get

$$
P^{*}=-2 \pi \int_{0}^{a} x(u, p) d u
$$

4. Results for special shapes of punches. We will now consider some special cases of the application of these formulae.

(a) Flat-ENDEd CYlindrical PUNCH. We begin by considering the case in which the half-space $z>0$ is deformed by the normal penetration of the boundary by a flatended rigid cylinder of radius $a$. We suppose that the punch penetrates a constant distance $u_{0}$. For this case, we assume that $f(r)=1, g(t)=1$. Using this, and upon nondimensionalizing equation (2.28) by the transformation of variables

$$
u=a u_{1}, \quad s=\frac{s_{1}}{a}, \quad P_{1}=\frac{a p}{k}, \quad \chi\left(a u_{1}, p\right)=\frac{u_{0} \Psi\left(u_{1}, P_{1}\right)}{\pi(1-v) K p},
$$


we find, from (3.1), that

$$
\Psi\left(u_{1}, P_{1}\right)+\int_{0}^{1} \Psi\left(v_{1}, P_{1}\right) M_{2}\left(u_{1}, v_{1}, P_{1}\right) d v_{1}=1, \quad 0<u_{1}<1,
$$

where

$$
\begin{aligned}
M_{2}\left(u_{1}, v_{1}, P_{1}\right) & =\frac{2}{\pi} \int_{0}^{\infty} K_{2}\left(s_{1}, P_{1}\right) \cos \left(u_{1} s_{1}\right) \cos \left(s_{1} v_{1}\right) d s_{1}, \\
K_{2}\left(s, P_{1}\right) & =K_{1}\left(s_{1}, P_{1}\right)-1, \\
K_{1}\left(s_{1}, P_{1}\right) & =\frac{\left(s_{1}^{2}+P_{1}\right)^{1 / 2}}{\left[2(1-v)\left(s_{1}^{2}+P_{1}\right)^{1 / 2}-(1-2 v) s_{1}\right]} .
\end{aligned}
$$

For this case, (3.4) can be written in the following form:

$$
P^{*}=-\frac{2 u_{0} a}{\alpha(1+v) p} \int_{0}^{1} \Psi\left(u_{1}, P_{1}\right) d u_{1}
$$

When $p \rightarrow 0$, we can easily find that

$$
P_{\infty}=(P)_{t \rightarrow \infty}=-\frac{2 a u_{0}}{(1+v) \alpha} .
$$

Making use of (4.4) and (4.5), we find that

$$
\frac{P^{*}}{P_{\infty}}=\frac{1}{p} \int_{0}^{1} \Psi\left(u_{1}, P_{1}\right) d u_{1} .
$$

We find that by using the inversion theorem of Laplace transforms,

$$
\frac{P}{P_{\infty}}=\frac{1}{2 \pi i} \int_{\beta_{r}} \frac{e^{P_{1} t_{1}} d P_{1}}{P_{1}}\left[\int_{0}^{1} \Psi\left(u_{1}, P_{1}\right) d u_{1}\right],
$$

where $\beta_{r}$ stands for a Bromwich path and

$$
t_{1}=\frac{k}{a} t
$$

Solving (4.2) numerically and then, from (4.7), by using the method of finding the inversion of Laplace transforms discussed by Miller and Guy [3], we find the numerical values of $P / P_{\infty}$. The results for $P / P_{\infty}$ against $t_{1}$ are shown in Figure 4.1 for the flatended cylindrical punch.

We can easily find from (2.10), (2.18), (2.22), (2.23), and (2.25) that

$$
\begin{aligned}
\frac{T^{*}}{c_{1}}= & \left(\frac{1-2 v}{1+v}\right) \int_{0}^{1} \Psi\left(u_{1}, P_{1}\right) d u_{1} \\
& \times \int_{0}^{\infty} \frac{e^{-\left(s_{1}^{2}+P_{1}\right) z_{1}} K_{1}\left(s_{1}, P_{1}\right) J_{0}\left(r_{1} s_{1}\right) \cos \left(s_{1} u_{1}\right) d s_{1}}{\left(s_{1}^{2}+P_{1}\right)^{1 / 2}},
\end{aligned}
$$

where

$$
r=a r_{1}, \quad z_{1}=\frac{z}{a}, \quad c_{1}=\frac{a u_{0}}{\pi \alpha k}
$$




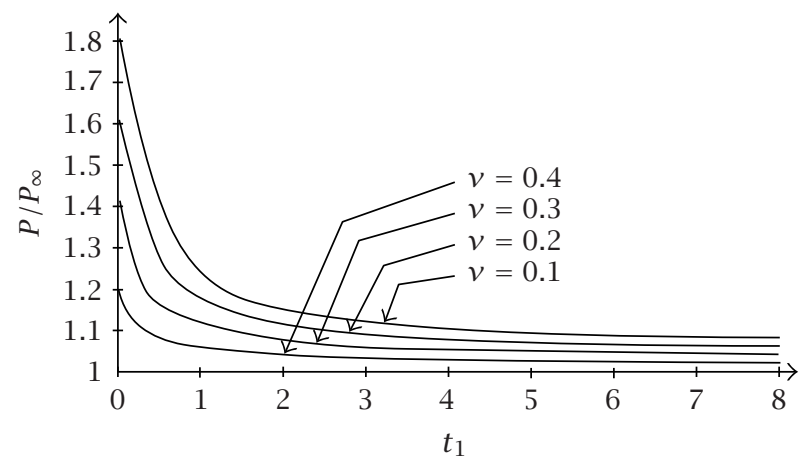

Figure 4.1. Variation of $P / P_{\infty}$ versus $t_{1}$ for values of $v=0.4,0.3,0.2,0.1$ and where $t_{1}=(k / a) t$ and $P_{\infty}=(P)_{t_{1} \rightarrow \infty}$.

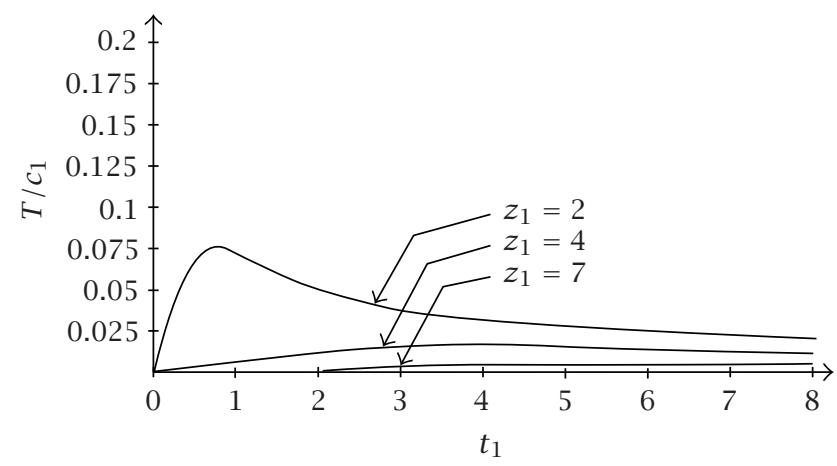

FiguRE 4.2. Variation of $T / c_{1}$ versus $t_{1}$ for values of $z_{1}=2,4,7$ and $r_{1}=1$.

Making use of (4.9) and the method of finding the inversion of Laplace transforms discussed in [3], we find the temperature field in the semi-infinite solid. The results are displayed in Figure 4.2.

(b) CONICAL PUNCH. For normal penetration by a rigid conical solid of semivertical angle $\alpha_{1}$, we may take $f(r)=\epsilon r$ and $g(t)=1$, where $\epsilon=\tan \alpha_{1}$. For this case, we find, from (2.28), that

$$
\Psi_{1}\left(u_{1}, P_{1}\right)+\int_{0}^{1} \Psi\left(v_{1}, P\right) M_{2}\left(u_{1}, v_{1}, P_{1}\right) d v_{1}=u_{1}, \quad 0<t_{1}<1,
$$

where

$$
\chi\left(a u_{1}, p\right)=\frac{a u_{0} \in \Psi_{1}\left(u_{1}, P_{1}\right)}{2 \alpha p(1+v)}
$$

For this case, (3.4) may be written in the following form:

$$
P^{*}=-\frac{\pi u_{0} a^{2} \epsilon}{(1+v) \alpha p} \int_{0}^{1} \Psi_{1}\left(u_{1}, P_{1}\right) d u_{1} .
$$




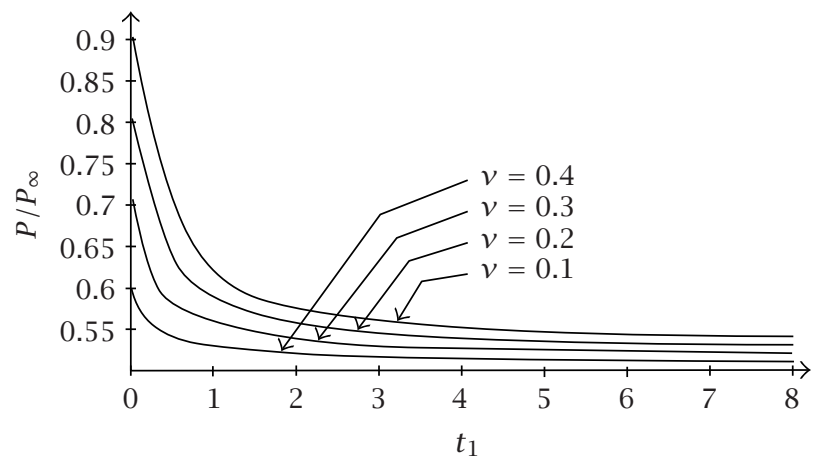

FIgURE 4.3. Variation of $P / P_{\infty}$ versus $t_{1}$ for values of $v=0.4,0.3,0.2,0.1$ and where $t_{1}=(k / a) t$ and $P_{\infty}=(P)_{t_{1} \rightarrow \infty}$.

When $p \rightarrow 0$, we find that

$$
P_{\infty}=(P)_{t \rightarrow \infty}=-\frac{a^{2} u_{0} \epsilon \pi}{2(1+v) \alpha} .
$$

Making use of (4.13) and (4.14), we find that

$$
\frac{P^{*}}{P_{\infty}}=\frac{2}{p} \int_{0}^{1} \Psi_{1}\left(u_{1}, P_{1}\right) d u_{1} .
$$

Now, by using the inversion theorem of Laplace transforms, we find that

$$
\frac{P}{2 P_{\infty}}=\frac{1}{2 \pi i} \int_{\beta_{r}} \frac{e^{P_{1} t_{1}} d P_{1}}{P_{1}} \int_{0}^{1} \Psi_{1}\left(u_{1}, P_{1}\right) d u_{1} .
$$

Solving (4.11) numerically and using (4.16) and the method of finding the inversion of Laplace transforms discussed by Miller and Guy [3], we find $P / 2(P)_{\infty}$. The results are displayed in Figure 4.3.

5. Conclusions. The numerical results of this work are displayed in Figures 4.1, 4.2, and 4.3. Figures 4.1 and 4.3 show the variation of the total load on the punch against $t_{1}$ for the cylindrical and conical punches, respectively. We notice from these figures that the total load on the punch decreases with time $t$ as well as Poisson's ratio $v$ of the material. And as $t_{1} \rightarrow 0$ or $t \rightarrow 0$, we find analytically that $P / P_{\infty} \rightarrow 2(1-v)$. For a cylindrical punch, we conclude, from Figure 4.2, that the temperature field decreases with the depth of the material.

\section{REFERENCES}

[1] J. Boussinesq, Application des Potentiels à l'Étude de l'Équilibre et du Mouvement des Solides Élastiques, Gauthier-Villars, Paris, 1985.

[2] L. A. Galin, Contact Problems of the Theory of Elasticity, Gosudarstv. Izdat. Tehn.-Teor. Lit., Moscow, 1953.

[3] M. K. Miller and W. T. Guy Jr., Numerical inversion of the Laplace transform by use of Jacobi polynomials, SIAM J. Numer. Anal. 3 (1966), 624-635. 
[4] I. N. Sneddon, Fourier Transforms, McGraw-Hill, New York, 1951.

[5] _ - The relation between load and penetration in the axisymmetric Boussinesa problem for a punch of arbitrary profile, Internat. J. Engrg. Sci. 3 (1965), 47-57.

[6] I. Todhunter and K. Pearson, A History of the Theory of Elasticity, Cambridge University Press, Cambridge, 1893.

J. Rokne: Department of Computer Science, University of Calgary, Calgary, Alberta, Canada T2N $1 \mathrm{~N} 4$

E-mail address: rokne@cpsc.ucalgary.ca

B. M. Singh: Department of Mathematics and Statistics, University of Calgary, Calgary, Alberta, Canada T2N $1 \mathrm{~N} 4$

R. S. Dhaliwal: Department of Mathematics and Statistics, University of Calgary, Calgary, Alberta, Canada T2N 1N4

E-mail address: dha7i . r@shaw.ca

J. Vrbik: Department of Mathematics, Brock University, St. Catharines, Ontario, Canada L2S 3A1 E-mail address: jvrbik@abacus.ac.brocku.ca 


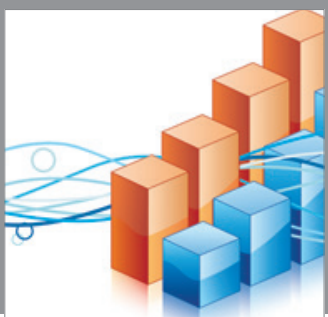

Advances in

Operations Research

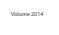

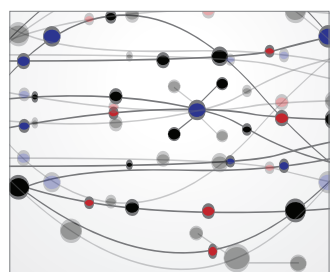

\section{The Scientific} World Journal
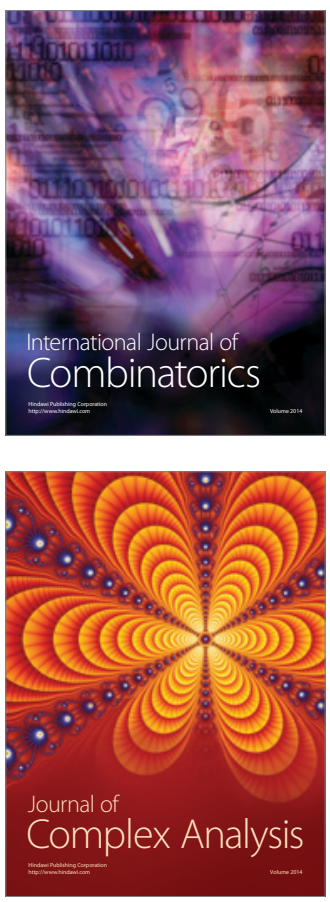

International Journal of

Mathematics and

Mathematical

Sciences
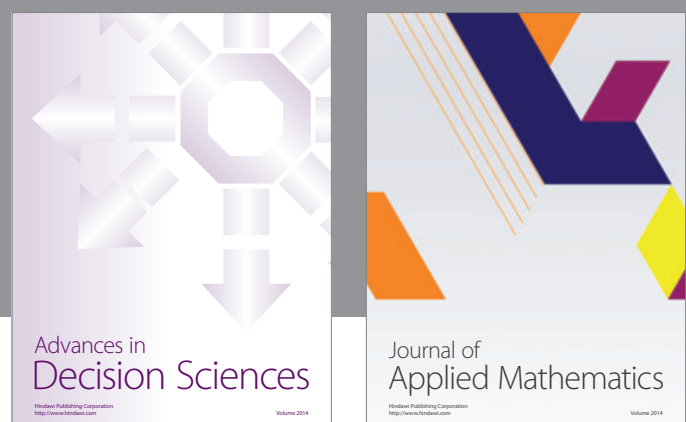

Journal of

Applied Mathematics
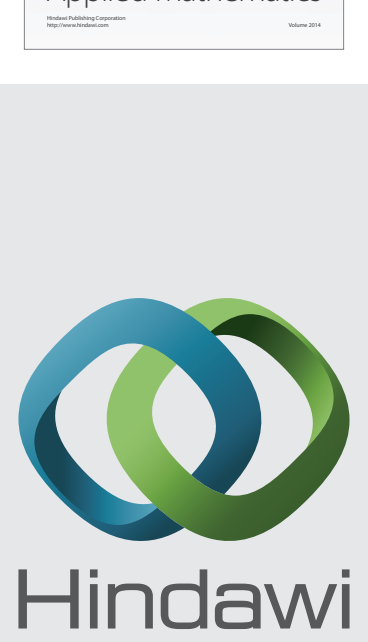

Submit your manuscripts at http://www.hindawi.com
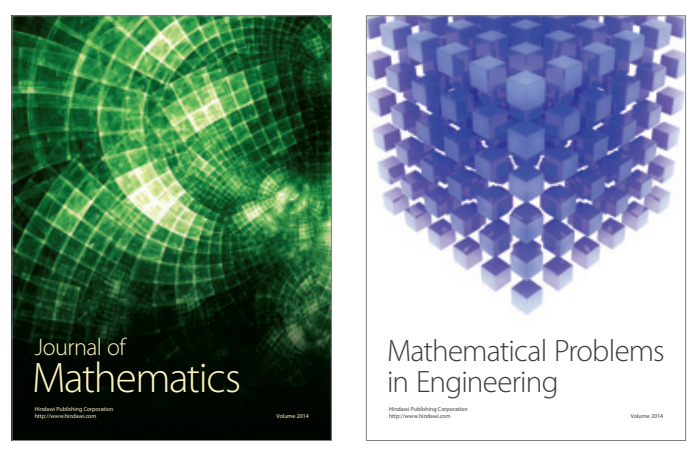

Mathematical Problems in Engineering
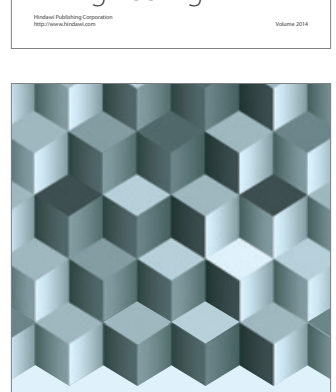

Journal of

Function Spaces
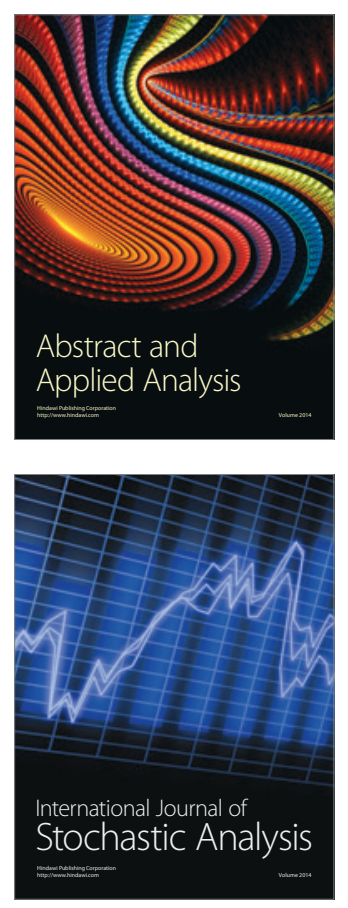

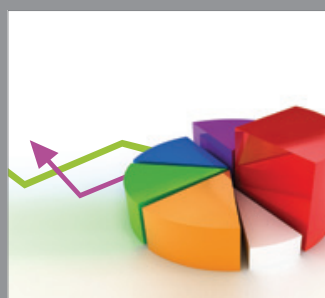

ournal of

Probability and Statistics

Promensencen
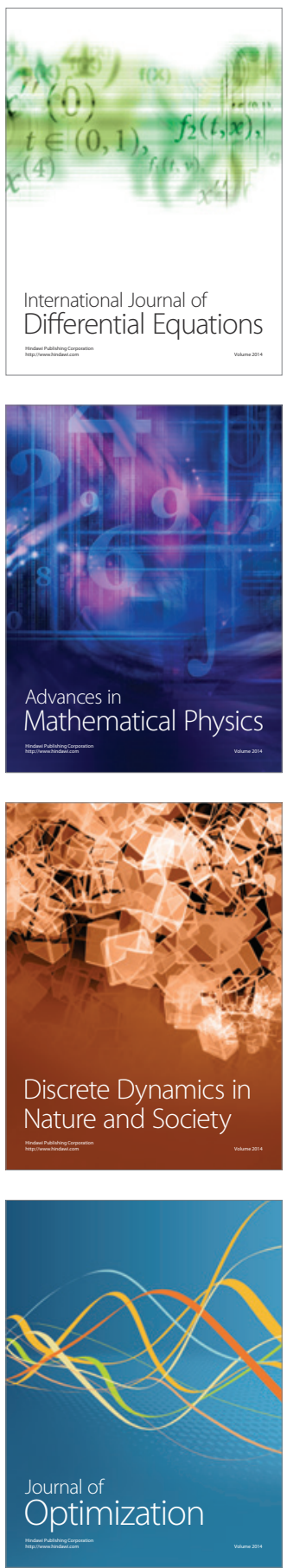